\title{
Long-term effects of multimodal treatment for patients with resectable carcinoma of the pancreas
}

\author{
TAKEHIRO OKABAYASHI ${ }^{1}$, ISAO NISHIMORI ${ }^{2}$, AKIHITO NISHIOKA $^{3}$, KOICHI YAMASHITA ${ }^{4}$, \\ TAKEKI SUGIMOTO ${ }^{1}$, KEN DABANAKA ${ }^{1}$, HIROMICHI MAEDA ${ }^{1}$, TAKUHIRO KOHSAKI ${ }^{2}$, \\ YASUHIRO OGAWA $^{3}$, MICHIYA KOBAYASHI ${ }^{5}$, SABURO ONISHI ${ }^{2}$ and KAZUHIRO HANAZAKI ${ }^{1}$ \\ Departments of ${ }^{1}$ Surgery, ${ }^{2}$ Gastroenterology and Hepatology, ${ }^{3}$ Diagnostic Radiology \\ and Radiation Oncology, ${ }^{4}$ Anesthesiology and Critical Care Medicine and ${ }^{5}$ Human Health and \\ Medical Services, Kochi Medical School, Kohasu-Okocho, Nankoku-City, Kochi 783-8505, Japan
}

Received March 3, 2008; Accepted May 19, 2008

DOI: 10.3892/or_00000055

\begin{abstract}
The treatment of pancreatic carcinoma remains one of the most formidable challenges in oncology. Curative resection, currently the only available treatment option, provides no significant impact on long-term survival. The recent development of multimodal treatment options for pancreatic cancer has provided clinical benefits and improved patient survival. In this study, we retrospectively evaluated our experiences with multimodal therapy, including radiotherapy and chemotherapy with gemcitabine, for the treatment of resectable pancreatic cancer. Fifty-eight patients with ordinary pancreatic carcinoma who underwent surgical resection at Kochi Medical School were studied. The clinical and pathological factors and multimodal treatment for pancreatic carcinoma that influenced patient survival were analyzed. Cumulative 1-, 3- and 5-year survival rates after surgery for ordinary pancreatic carcinoma were $62.2,20.3$ and $20.3 \%$, respectively. The overall 4-year survival rate of patients subjected to adjuvant chemotherapy with gemcitabine after curative resection for ordinary pancreatic carcinoma is $39.1 \%$. Adjuvant chemotherapy with gemcitabine provided a significantly better prognosis for patients following curative surgical resection than curative surgical resection alone $(\mathrm{P}=0.035)$. Although the rate of survival was greater for patients who underwent radiotherapy than those who did not, the difference was not statistically significant $(\mathrm{P}=0.054)$. Postoperative local recurrence around the nerve plexus of celiac and superior mesenteric arteries was better controlled in patients who underwent radiotherapy than those who did not. Adjuvant chemotherapy with gemcitabine after curative
\end{abstract}

Correspondence to: Dr Takehiro Okabayashi, Department of Surgery, Kochi Medical School, Kohasu-Okocho, Nankoku-City, Kochi 783-8505, Japan

E-mail: tokabaya@kochi-u.ac.jp

Key words: pancreas, pancreatic carcinoma, chemotherapy, surgery, radiotherapy resection provides a significant survival benefit for patients with pancreatic carcinoma. Our results suggest that the postoperative recurrence of ordinary pancreatic carcinoma will be reduced by multimodal treatment using radiotherapy and adjuvant chemotherapy with gemcitabine.

\section{Introduction}

Pancreatic carcinoma is a near fatal disease and one of the most aggressive human malignancies (1-3). Pancreatectomy with regional lymphadenectomy is the only curative treatment option for pancreatic cancer, although the extent of lymphadenectomy is of no clinical benefit according to randomized trial studies (4). When surgery is a treatment option for pancreatic cancer, the 5-year survival rate rises to only $7.4-13.4 \%$ (5-7). This poor survival rate is attributed to a high incidence of local recurrence and development of distant metastases.

The recent development of multimodal treatment options for pancreatic cancer, including radiotherapy and chemotherapy with gemcitabine, is reported to provide clinical benefits and improvements in survival (8-10). Multimodal therapy is proposed to reduce local and systemic recurrence of pancreatic cancer and improve survival, although the benefit of adjuvant therapy is not fully accepted in the medical community (11-13). In the present study, we retrospectively evaluated multimodal therapy for the treatment of resectable pancreatic cancer.

\section{Patients and methods}

A total of 58 patients who underwent surgery as an initial treatment for pancreatic carcinoma between June 1982 and July 2007 at Kochi Medical School were studied. Of these patients, 25 were men and 33 were women, ranging in age from 35 to 81 years (mean 67.3). Curative resection was the operative aim for all patients.

Clinical characteristics were evaluated for age, gender, body mass index (BMI), preoperative laboratory investigations, past medical history for diabetes mellitus and vascular diseases, pancreatitis, operation time, estimated blood loss, 
Table I. Clinicopathological features of patients with pancreatic carcinoma.

\begin{tabular}{|c|c|c|c|c|c|}
\hline \multirow[b]{2}{*}{ Characteristics } & \multicolumn{2}{|c|}{ Gemcitabine (-) } & \multicolumn{2}{|c|}{ Gemcitabine (+) } & \multirow[b]{2}{*}{ P-value } \\
\hline & $\begin{array}{l}\mathrm{RT}(-) \\
(\mathrm{n}=18)\end{array}$ & $\begin{array}{l}\mathrm{RT}(+) \\
(\mathrm{n}=13)\end{array}$ & $\begin{array}{l}\text { RT (-) } \\
(\mathrm{n}=6)\end{array}$ & $\begin{array}{l}\mathrm{RT}(+) \\
(\mathrm{n}=21)\end{array}$ & \\
\hline Age & $68 \pm 9$ & $60 \pm 11^{\mathrm{a}}$ & $71 \pm 7$ & $70 \pm 7$ & SD \\
\hline Gender (male/female) & $7 / 11$ & $8 / 5$ & $4 / 2$ & $6 / 15$ & NS \\
\hline Body mass index & $23.1 \pm 3.0$ & $20.1 \pm 2.8^{\mathrm{b}}$ & $22.8 \pm 2.7$ & $21.3 \pm 3.0$ & SD \\
\hline Diabetes mellitus (yes/no) & $12 / 6$ & $8 / 5$ & $5 / 1$ & $15 / 6$ & NS \\
\hline Pancreatitis (yes/no) & $7 / 8$ & $2 / 11$ & $4 / 2$ & $5 / 16$ & NS \\
\hline Total protein & $6.6 \pm 0.6$ & $6.7 \pm 0.7$ & $6.6 \pm 0.4$ & $7.2 \pm 0.6^{c}$ & SD \\
\hline Albumin & $3.8 \pm 0.5$ & $4.0 \pm 0.7$ & $3.9 \pm 0.4$ & $4.3 \pm 0.5$ & NS \\
\hline Fasting blood sugar & $129 \pm 67$ & $141 \pm 48$ & $137 \pm 54$ & $137 \pm 44$ & NS \\
\hline Cholinesterase & $223 \pm 60$ & $232 \pm 94$ & $180 \pm 33$ & $268 \pm 58^{\mathrm{d}}$ & $\mathrm{SD}$ \\
\hline Amylase & $293 \pm 345$ & $106 \pm 64$ & $94 \pm 32$ & $108 \pm 136$ & NS \\
\hline Carcinoembryonic antigen & $4.1 \pm 8.1$ & $4.6 \pm 3.7$ & $2.4 \pm 0.4$ & $11.7 \pm 30.0$ & NS \\
\hline Carbohydrate antigen 19-9 & $930 \pm 1381$ & $166 \pm 218$ & $87 \pm 84$ & $371 \pm 558$ & NS \\
\hline $\begin{array}{l}\text { Location of the tumor } \\
\text { Head/body/tail }\end{array}$ & $10 / 4 / 4$ & $10 / 3 / 0$ & $4 / 1 / 1$ & $18 / 2 / 1$ & NS \\
\hline Size of the tumor $(\mathrm{cm})$ & $4.0 \pm 2.0$ & $3.6 \pm 1.3$ & $4.2 \pm 2.9$ & $3.0 \pm 0.7$ & NS \\
\hline
\end{tabular}

RT, radiotherapy; SD, significant difference and NS, not significant. ${ }^{a}$ The 13 patients subjected to radiotherapy alone were significantly younger than other groups $(\mathrm{P}=0.001)$. ${ }^{\mathrm{B}} \mathrm{BMI}$ was significantly reduced in the group of 13 patients subjected to radiotherapy alone than in the group of 18 patients not subjected to adjuvant therapy $(\mathrm{P}=0.017)$. ${ }^{\mathrm{c}}$ Maintenance of the preoperative total protein levels was significant in the 21 patients subjected to both radiotherapy and chemotherapy and not in the other groups $(\mathrm{P}=0.002)$. ${ }^{\mathrm{d}}$ Maintenance of the preoperative cholinesterase levels was significant in the 21 patients subjected to radiotherapy and chemotherapy and not in the other groups $(\mathrm{P}=0.009)$.

operative procedures, intra-operative radiotherapy and postoperative chemotherapy. The BMI was calculated by division of weight in kilograms by the square of height in meters. Location of pancreatic cancer, size of the tumor, stage, degree of differentiation, vascular invasion, lymphatic permeation and lymph node metastasis were assessed according to the guidelines of the American Joint Committee on Cancer (14). Our department followed the prognosis of each case and obtained accurate details of outcome. In this series, we evaluated ordinary invasive ductal carcinoma of the pancreas and excluded the invasive pancreatic carcinoma derived from intraductal papillary mucinous neoplasm.

Of the 58 patients included in the study, 25 underwent pancreaticoduodenectomy, 17 underwent pylorus-preserving pancreaticoduodenectomy and 16 underwent distal pancreatectomy and splenectomy. Of the 58 patients with resectable pancreatic carcinoma, 34 patients received intra-operative radiotherapy with $12 \mathrm{MeV}$ electron beams. The intra-operative field set by the use of treatment applications of $8 \mathrm{~cm}$ in diameter covered the tumor bed and a margin of $>1 \mathrm{~cm}$. The intra-operative radiotherapy dose was $20 \mathrm{~Gy}$. We included the resected edge of the pancreas within the intra-operative radiotherapy field. External beam radiotherapy following intra-operative radiotherapy was initiated routinely after April 2003. External beam radiotherapy, at a dose of $40 \mathrm{~Gy}$, was performed according to the patients' postoperative condition ( $\mathrm{n}=17)$. Adjuvant chemotherapy comprising of a drip infusion of gemcitabine commenced at the time of external beam radiotherapy.

Statistical analysis. Clinicopathological findings were compared using the t-test, Pearson Chi-square test and Aspin-Welch test. Where appropriate, values were expressed as mean \pm standard deviation. Survival rates were generated using the Kaplan-Meier method and were compared using the log-rank test (15). A value of $\mathrm{P}<0.05$ was considered significant.

\section{Results}

There was no mortality in this series. The clinical characteristics of pancreatic carcinoma are shown in Table I. The 58 patients consisted of 18 not subjected to adjuvant chemotherapy with gemcitabine, 13 subjected to radiotherapy alone, 6 subjected to adjuvant chemotherapy with gemcitabine alone and 21 subjected to radiotherapy and adjuvant chemotherapy with gemcitabine. There was no significant difference among the four groups with respect to gender, past medical history for diabetes mellitus and pancreatitis and the clinical features of patients with resectable pancreatic carcinoma. The mean age of the four groups was $68 \pm 9,60 \pm 11$, $71 \pm 7$ and $70 \pm 7$ years, respectively. The 13 patients who 
Table II. Operative characteristics and pathological features of pancreatic carcinoma.

\begin{tabular}{|c|c|c|c|c|c|}
\hline \multirow[b]{2}{*}{ Characteristics } & \multicolumn{2}{|c|}{ Gemcitabine (-) } & \multicolumn{2}{|c|}{ Gemcitabine (+) } & \multirow[b]{2}{*}{ P-value } \\
\hline & $\begin{array}{l}\mathrm{RT}(-) \\
(\mathrm{n}=18)\end{array}$ & $\begin{array}{l}\mathrm{RT}(+) \\
(\mathrm{n}=13)\end{array}$ & $\begin{array}{c}\text { RT (-) } \\
(\mathrm{n}=6)\end{array}$ & $\begin{array}{l}\mathrm{RT}(+) \\
(\mathrm{n}=21)\end{array}$ & \\
\hline \multicolumn{6}{|l|}{ Operative procedures } \\
\hline DHP/DPS & $10 / 8$ & $10 / 3$ & $4 / 2$ & $18 / 3$ & NS \\
\hline Operation time & $455 \pm 125$ & $393 \pm 119$ & $384 \pm 174$ & $485 \pm 110$ & NS \\
\hline Estimated blood loss & $1288 \pm 1044$ & $1117 \pm 658$ & $947 \pm 588$ & $726 \pm 284$ & NS \\
\hline Cumulative stage & $6 / 7 / 5$ & $2 / 9 / 2$ & $1 / 4 / 1$ & $9 / 9 / 3$ & NS \\
\hline
\end{tabular}

DHP, duodenohemipancreatectomy, including pancreaticoduodenectomy and pylorus-preserving pancreaticoduodenectomy. DPS, distal pancreatectomy with splenectomy and RT, radiotherapy.

underwent radiotherapy alone were significantly younger than the patients in the other three groups. Patients in the radiotherapy alone group also had a significantly lower BMI than the 18 patients of the no adjuvant chemotherapy with gemcitabine group $(\mathrm{P}=0.017)$. The maintenance of preoperative total protein and cholinesterase levels was significant for the 21 patients in the radiotherapy and adjuvant chemotherapy with gemcitabine group and not for patients in the other three groups. Other preoperative serum chemistry, including levels of albumin, fasting blood sugar, amylase, carcinoembryonic antigen, carbohydrate antigen 19-9, were not significantly different between the four groups. The majority of the tumors were situated at the head of the pancreas. The size of the tumors was $4.0 \mathrm{~cm}$ in the no adjuvant chemotherapy with gemcitabine group, $3.6 \mathrm{~cm}$ in the group subjected to radiotherapy alone, $4.2 \mathrm{~cm}$ in the group subjected to adjuvant chemotherapy with gemcitabine alone and $3.0 \mathrm{~cm}$ in the group subjected to radiotherapy and adjuvant chemotherapy with gemcitabine. There was no significant difference between the four groups in the localization of the pancreatic carcinoma and the size of the tumor.

The type of operation was not significantly different between the four groups; duodenohemipancreatectomy was performed in $55.6 \%$ of patients not subjected to adjuvant chemotherapy with gemcitabine, $76.9 \%$ of patients subjected to radiotherapy alone, $66.7 \%$ of patients subjected to adjuvant chemotherapy with gemcitabine alone and $85.7 \%$ of patients subjected to radiotherapy and adjuvant chemotherapy with gemcitabine. The operation time was $455 \mathrm{~min}$ for patients subjected to no adjuvant chemotherapy with gemcitabine, 393 for those subjected to radiotherapy alone, 384 for those subjected to adjuvant chemotherapy with gemcitabine alone and 485 for those subjected to radiotherapy and adjuvant chemotherapy with gemcitabine. The estimated blood loss volume was not significantly different between the four groups (Table II). There was no significant difference between the four groups in the conclusive curability, pathological stage, or degree of differentiation of the pancreatic carcinoma.

Of the 58 patients, 17 (29.3\%) showed no recurrence of cancer. The major sites of recurrence were the liver (26 patients), peritoneum (11 patients) and local nerve plexus

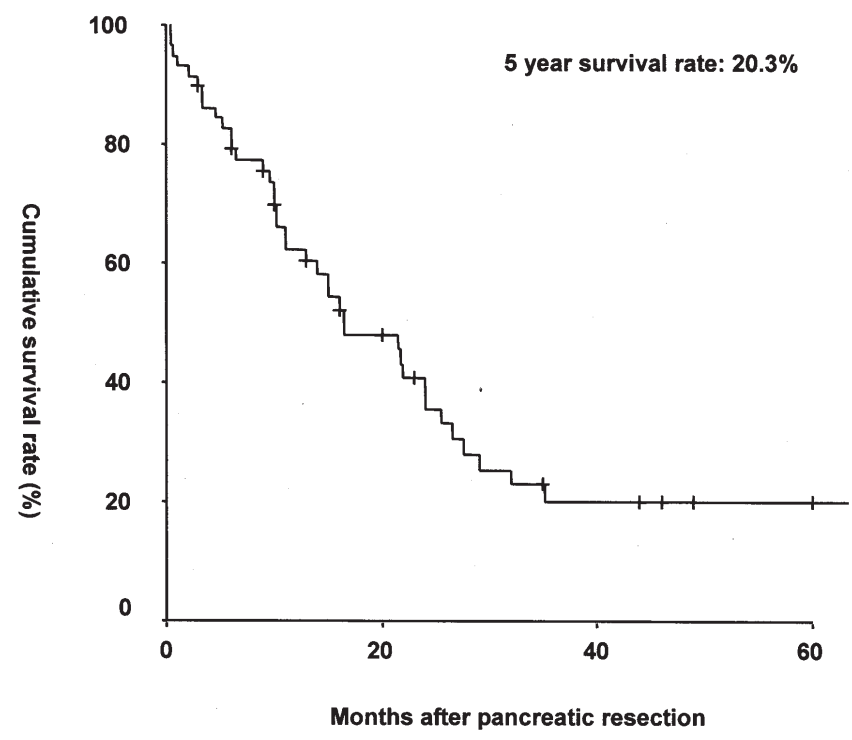

Figure 1. Survival curves for the 58 patients. Cumulative 1-, 3- and 5-year survival rates after surgery for ordinary pancreatic carcinoma in the current study were $62.2,20.3$ and $20.3 \%$, respectively.

around the superior mesenteric artery (10 patients) (Table III). No recurrence rate $(14 / 27)$ for patients to gemcitabine was significantly less than the recurrence rate observed for patients not subjected to gemcitabine $(3 / 31)(\mathrm{P}=0.001)$. With respect to multimodal treatment, the overall recurrence rate of $38.1 \%$ for patients subjected to radiotherapy and adjuvant chemotherapy with gemcitabine was significantly less than the recurrence rate of $89.2 \%$ observed for patients not subjected to multimodal treatment $(\mathrm{P}<0.001)$. The local recurrence rate of cancer after surgery for patients not subjected to radiotherapy was $33.3 \%$. This is significantly greater than the $15.0 \%$ local recurrence rate observed for patients not subjected to radiotherapy $(\mathrm{P}=0.075)$. The site of cancer recurrence did not differ between patients subjected to and not subjected to radiotherapy for ordinary pancreatic carcinoma. Postoperative local recurrence around the nerve plexus of celiac and superior mesenteric arteries was better controlled in patients subjected to external beam radiotherapy following intra-operative 
Table III. Pattern of recurrence after surgery.

\begin{tabular}{|c|c|c|c|c|c|}
\hline \multirow[b]{2}{*}{ Characteristics } & \multicolumn{2}{|c|}{ Gemcitabine (-) } & \multicolumn{2}{|c|}{ Gemcitabine (+) } & \multirow[b]{2}{*}{ P-value } \\
\hline & $\begin{array}{l}\text { RT (-) } \\
(\mathrm{n}=16)\end{array}$ & $\begin{array}{l}\mathrm{RT}(+) \\
(\mathrm{n}=12)\end{array}$ & $\begin{array}{c}\text { RT (-) } \\
(\mathrm{n}=5)\end{array}$ & $\begin{array}{c}\mathrm{RT}(+) \\
(\mathrm{n}=8)\end{array}$ & \\
\hline Liver only & 5 & 4 & 3 & 4 & NS \\
\hline $\begin{array}{l}\text { Liver }+ \\
\text { Peritoneum } \\
\text { Local }\end{array}$ & $\begin{array}{l}3 \\
1\end{array}$ & $\begin{array}{l}4 \\
0\end{array}$ & $\begin{array}{l}0 \\
1\end{array}$ & $\begin{array}{l}1 \\
0\end{array}$ & NS \\
\hline Local & 5 & 2 & 0 & 1 & NS \\
\hline Peritoneum & 1 & 1 & 0 & 0 & NS \\
\hline Lung & 1 & 1 & 0 & 1 & NS \\
\hline Others & 0 & 0 & 1 & 1 & NS \\
\hline No recurrence & $2 / 18$ & $1 / 13$ & $1 / 6$ & $13 / 21$ & 0.001 \\
\hline
\end{tabular}

RT, radiotherapy.



Months after pancreatic resection

Figure 2. Survival curve for patients in the presence and absence of multimodal treatment for ordinary pancreatic carcinoma. (a) Patients undergoing curative surgical resection with adjuvant chemotherapy using gemcitabine for ordinary pancreatic carcinoma had a significantly better outcome than patients undergoing potentially curative resection without adjuvant chemotherapy by gemcitabine for pancreatic carcinoma ( $\mathrm{P}=0.035)$. (b) The survival curve for patients subjected to radiotherapy tended to be more favorable than for patients not subjected to radiotherapy; however there was no statistically significant difference ( $\mathrm{P}=0.054$ ). Gem, gemcitabine and RT, radiotherapy.

radiotherapy after curative resection $(\mathrm{n}=17)$ than in patients not subjected to radiotherapy ( $\mathrm{P}=0.044$, Pearson correlation value).

Fig. 1 shows the survival curves for the 58 patients. At the time of the present study, 17 patients were alive with four surviving for $>5$ years after surgery. Cumulative 1-, 3- and 5 -year survival rates after surgery for ordinary pancreatic carcinoma in the current study were $62.2,20.3$ and $20.3 \%$, respectively. Fig. 2 shows the survival curves for patients subjected to and not subjected to multimodal treatment for ordinary pancreatic carcinoma. The patient group treated with adjuvant chemotherapy using gemcitabine following curative surgical resection for ordinary pancreatic carcinoma had a 4-year cumulative survival rate of $39.1 \%$ and a median survival period of 24 months. By comparison, the patient group not treated using adjuvant chemotherapy with gemcitabine following curative resection had a median survival period of 13 months and a 4- and 5-year cumulative survival rate of $12.5 \%(\mathrm{P}=0.035)$. This result shows that adjuvant chemotherapy with gemcitabine for ordinary pancreatic carcinoma provides a significantly improved survival outcome following curative surgical resection. Although the survival curve was more favorable for patients subjected to radiotherapy than not, the difference is not statistically significant $(\mathrm{P}=0.054)$. 


\section{Discussion}

Pancreatic carcinoma is the most aggressive malignancy of the gastrointestinal tract. The reported incidence of ordinary pancreatic carcinoma has gradually increased with advances in diagnostic techniques and equipment. Surgical treatment remains the only curative management option for ordinary pancreatic carcinoma. Extended lymphadenectomy in pancreaticoduodenectomy for pancreatic carcinoma does not benefit overall survival and prospective trials indicate a trend towards increased morbidity $(16,17)$. Extended aggressive surgery for distal pancreatectomy has also been attempted (18). Standard pancreatectomy with regional lymphadenectomy for ordinary pancreatic carcinoma was performed in our department. Limitations of the present study include the associated errors and biases inherent in a small retrospective study design. We showed that adjuvant chemotherapy using gemcitabine may not only improve the long-term survival of patients subjected to curative resection for pancreatic carcinoma, but also reduce postoperative recurrence when used in combination with radiotherapy.

Adjuvant chemotherapy is the standard treatment following resection for pancreatic carcinoma. Recent reports suggest that adjuvant chemotherapy with gemcitabine provides a significant increase in patient survival $(9,19,20)$. In a randomized control trial, Oettle et al (21) reported a disease-free survival rate 3 years following surgery of $23.5 \%$ for patients treated with adjuvant chemotherapy with gemcitabine and $7.5 \%$ in the control group receiving no postoperative treatment. The post-operative administration of gemcitabine significantly delayed the development of recurrent disease after complete resection of pancreatic cancer compared with observation alone; however, there was no significant difference in overall survival between the gemcitabine group and the control group of patients (21). In the present study, patients who received adjuvant chemotherapy with gemcitabine after surgery were managed by an oncology specialist. Our study reports an overall 4-year survival rate of $39.1 \%$ for patients receiving postoperative adjuvant chemotherapy with gemcitabine. The cumulative survival of patients undergoing curative resection combined with adjuvant chemotherapy with gemcitabine (4-year survival rate: $39.1 \%$ ) was significantly greater than those undergoing curative resection alone (4-year survival rate: $12.5 \%$ ) (Fig. $2 \mathrm{a}$ ). We showed a significant reduction in the postoperative recurrence of disease, including liver metastases, peritoneal dissemination and/or local site recurrence $(\mathrm{P}<0.001)$, when adjuvant chemotherapy with gemcitabine is used to treat patients following curative resection for pancreatic carcinoma and suggested this to be the standard treatment option.

Evidence that adjuvant chemoradiotherapy for pancreatic carcinoma improves local control is inconclusive and better local control has not been shown to correlate with increased survival (22). While extended resection or radiotherapy is a treatment option for the local control of ordinary pancreatic carcinoma, extended resection, typically performed with retroperitoneal lymphadenectomy and circumferential clearance of the celiac and superior mesenteric arteries, has been associated with an increase in postoperative morbidity and no improvement in survival $(17,23)$. Adverse findings in some patients following radical resection for pancreatic carcinoma include impaired quality of life, postoperative diarrhea, body weight loss and nutritional disturbances. Two retrospective studies investigating the benefit of radiotherapy following curative resection for pancreatic carcinoma showed no significant difference in overall survival between patients treated or not treated with radiotherapy $(13,24)$. There are no published prospective randomized trials investigating radiotherapy for the treatment of pancreatic carcinoma following curative resection. In the present study, postoperative local recurrence around the nerve plexus of celiac and superior mesenteric arteries was better controlled in patients subjected to external beam radiotherapy following intra-operative radiotherapy after standard pancreatic resection than those not subjected to radiotherapy $(\mathrm{P}=0.044)$. Although radiotherapy did not benefit cumulative survival (Fig. 2b), the overall recurrence rate of $38.1 \%$ for patients subjected to multimodal treatment of radiotherapy and adjuvant chemotherapy with gemcitabine was significantly better than the recurrence rate of $89.2 \%$ observed for patients not subjected to multimodal treatment (Table III).

Malabsorption and maldigestion of nutrients induces severe malnutrition in patients with pancreatic cancer. An appropriate calorie intake is reported to prolong the survival of patients with advanced pancreatic cancer and to achieve a beneficial nutritional status related outcome for patients with pancreatic cancer $(25,26)$. In the present study, patients subjected to adjuvant chemotherapy with gemcitabine had a significantly better preoperative nutritional status, according to BMI and laboratory investigations, including body total protein and cholinesterase, than patients not subjected to adjuvant chemotherapy with gemcitabine. These results suggest that cumulative survival is significantly better for patients subjected to adjuvant chemotherapy with gemcitabine for resectable pancreatic cancer in the absence of symptoms of malnutrition.

In conclusion, adjuvant chemotherapy with gemcitabine after curative resection has a significant survival benefit for patients with pancreatic carcinoma. Our results suggest that overall recurrence after curative resection for ordinary pancreatic carcinoma can be reduced by multimodal treatment involving radiotherapy and adjuvant chemotherapy with gemcitabine.

\section{References}

1. Brunner TB, Grabenbauer GG, Meyer T, Golcher H, Sauer R and Hohenberger W: Primary resection versus neoadjuvant chemoradiation followed by resection for locally resectable or potentially resectable pancreatic carcinoma without distant metastasis. A multi-centre prospectively randomised phase IIstudy of the Interdisciplinary Working Group Gastrointestinal Tumours (AIO, ARO, and CAO). BMC Cancer 7: 41, 2007.

2. Palmer DH, Stocken DD, Hewitt H, Markham CE, Hassan AB, Johnson PJ, Buckels JA and Bramhall SR: A randomized phase 2 trial of neoadjuvant chemotherapy in resectable pancreatic cancer: gemcitabine alone versus gemcitabine combined with cisplatin. Ann Surg Oncol 14: 2088-2096, 2007.

3. Kleeff J, Michalski CW, Friess H and Büchler MW: Surgical treatment of pancreatic cancer: the role of adjuvant and multimodal therapies. Eur J Surg Oncol 33: 817-823, 2007.

4. Michalski CW, Kleeff J, Wente MN, Diener MK, Büchler MW and Friess H: Systematic review and meta-analysis of standard and extended lymphadenectomy in pancreaticoduodenectomy for pancreatic cancer. Br J Surg 94: 265-273, 2007. 
5. Nitecki SS, Sarr MG, Colby TV and van Heerden JA: Long-term survival after curative resection for ductal adenocarcinoma of the pancreas. Is it really improving? Ann Surg 221: 59-66, 1995.

6. Matsuno S, Egawa S, Fukuyama S, Motoi F, Sunamura M, Isaji S, Imaizumi T, Okada S, Kato H, Suda K, Nakao A, Hiraoka T, Hosotani R and Takeda K: Pancreatic Cancer Registry in Japan: 20 years of experience. Pancreas 28: 219-230, 2004.

7. Nakao A, Fujii T, Sugimoto H, Kanazumi N, Nomoto S, Kodera Y, Inoue $S$ and Takeda $S$ : Oncological problem in pancreatic cancer surgery. World J Gastroenterol 12: 4466-4472, 2006.

8. Fujino Y, Ueda T, Kamigaki T, Takase S, Ajiki T, Kamoda Y, Matsumoto I, Yasuda T and Kuroda Y: Impact of gemcitabine on the survival of patients with stage IV pancreatic cancer. Pancreas 34: 335-339, 2007.

9. O'Connor JK, Sause WT, Hazard LJ, Belnap LP and Noyes RD: Survival after attempted surgical resection and intraoperative radiation therapy for pancreatic and periampullary adenocarcinoma. Int J Radiat Oncol Biol Phys 63: 1060-1066, 2005.

10. Yamaguchi K, Nakamura K, Kobayashi K, Nakano K, Konomi H, Mizumoto K and Tanaka M: ERT following IORT improves survival of patients with resectable pancreatic cancer. Hepatogastroenterology 52: 1244-1249, 2005.

11. Demols A, Peeters M, Polus M, Honoré P, Boterberg T, Gay F, Closon MT, Van Houtte P, Closset J and Van Laethem JL: Adjuvant gemcitabine and concurrent continuous radiation (45 Gy) for resected pancreatic head carcinoma: a multicenter Belgian Phase II study. Int J Radiat Oncol Biol Phys 62: 1351-1356, 2005.

12. Willett CG, Czito BG and Bendell JC: Adjuvant therapy of pancreatic cancer. Cancer J 13: 185-191, 2007.

13. Ihse I, Andersson R, Ask A, Ewers SB, Lindell G and Tranberg KG: Intraoperative radiotherapy for patients with carcinoma of the pancreas. Pancreatology 5: 438-442, 2005.

14. Exocrine pancreas. In: American Joint Committee on Cancer: AJCC Cancer Staging Manual. 5th edition, Lippincott-Raven Publishers, Philadelphia, pp121-126, 1997.

15. Kaplan EL and Meier P: Nonparametric estimation from incomplete observations. J Am Stat Assoc 53: 457, 1958.

16. Farnell MB, Pearson RK, Sarr MG, DiMagno EP, Burgart LJ, Dahl TR, Foster N and Sargent DJ: Pancreas Cancer Working Group. A prospective randomized trial comparing standard pancreatoduodenectomy with pancreatoduodenectomy with extended lymphadenectomy in resectable pancreatic head adenocarcinoma. Surgery 138: 618-628, 2005.

17. Yeo CJ, Cameron JL, Lillemoe KD, Sohn TA, Campbell KA, Sauter PK, Coleman J, Abrams RA and Hruban RH: Pancreaticoduodenectomy with or without distal gastrectomy and extended retroperitoneal lymphadenectomy for peri-ampullary adenocarcinoma, part 2: randomized controlled trial evaluating survival, morbidity, and mortality. Ann Surg 236: 355-366, 2002.
18. Hirano S, Kondo S, Hara T, Ambo Y, Tanaka E, Shichinohe T, Suzuki O and Hazama K: Distal pancreatectomy with en bloc celiac axis resection for locally advanced pancreatic body cancer: long-term results. Ann Surg 246: 46-51, 2007.

19. Neoptolemos JP, Dunn JA, Stocken DD, Almond J, Link K, Beger H, Bassi C, Falconi M, Pederzoli P, Dervenis C, Fernandez-Cruz L, Lacaine F, Pap A, Spooner D, Kerr DJ, Friess $\mathrm{H}$ and Büchler MW: European study group for pancreatic cancer. Adjuvant chemoradiotherapy and chemotherapy in resectable pancreatic cancer: a randomised controlled trial. Lancet 358: 1576-1585, 2001.

20. Neuhaus P, Oettle H, Post S, Gellert K, Ridwelski K, Schramm H, Zurke C, Fahlke G, Langrehr J and Reiss H: A randomized, postoperative, multicenter, phase III trial of adjuvant chemotherapy with gemcitabine vs. observation in patients with resected pancreatic cancer. Proc Am Soc Clin Oncol 24: 4013, 2005.

21. Oettle H, Post S, Nauhasu P, Gellert K, Langrehr J, Ridwelski K, Schramm H, Fahlke J, Zuelke C, Burkart C, Gutberlet K, Kettner E, Schmanlenberg H, Weigang-Koehler K, Bechstein WO, Niedergethmann M, Schmidt-Wolf I, Roll L, Doerken B and Riess H: Adjuvant chemotherapy with gemcitabine vs observation in patients undergoing curative-intent resection of pancreatic cancer: a randomized control trial. JAMA 297: 267$277,2007$.

22. Neoptolemos JP, Stocken DD, Friess H, Bassi C, Dunn JA, Hickey H, Beger H, Fernandez-Cruz L, Dervenis C, Lacaine F, Falconi M, Pederzoli P, Pap A, Spooner D, Kerr DJ and Büchler MW: European study group for pancreatic cancer. A randomized trial of chemoradiotherapy and chemotherapy after resection of pancreatic cancer. N Engl J Med 350: 1200-1210, 2004.

23. Nguyen TC, Sohn TA, Cameron JL, Lillemoe KD, Campbell KA, Coleman J, Sauter PK, Abrams RA, Hruban RH and Yeo CJ: Standard vs. radical pancreaticoduodenectomy for periampullary adenocarcinoma: a prospective, randomized trial evaluating quality of life in pancreaticoduodenectomy survivors. J Gastrointest Surg 7: 1-11, 2003.

24. Nakagohri T, Kinoshita T, Konishi M, Takahashi S and Tanizawa Y: Clinical results of extended lymphadenectomy and intraoperative radiotherapy for pancreatic adenocarcinoma. Hepatogastroenterology 54: 564-569, 2007.

25. Okusaka T, Okada S, Ishii H, Ikeda M, Kosakamoto H and Yoshimori M: Prognosis of advanced pancreatic cancer patients with reference to calorie intake. Nutr Cancer 32: 55-58, 1998.

26. Bauer J, Capra S, Battistutta D, Davidson W and Ash S: Cancer cachexia study group. Compliance with nutrition prescription improves outcomes in patients with unresectable pancreatic cancer. Clin Nutr 24: 998-1004, 2005. 